\title{
Numerical Simulation of the Longitudinal Movement of a SSB Catamaran in Regular Waves
}

\author{
HanBing Sun ${ }^{1, a}$, Yi Jiang ${ }^{1, b}$, JiaYuan Zhuang ${ }^{1, c^{*}}$, Jin Zou ${ }^{1}$ \\ ${ }^{1}$ College of shipbuilding Engineering, Harbin Engineering University, Harbin, 150001, China

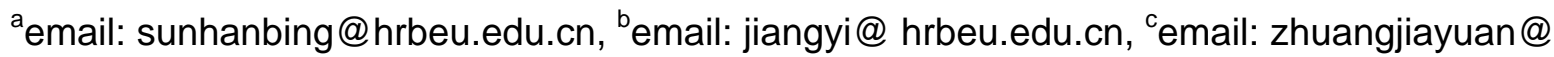 \\ hrbeu.edu.cn
}

Keywords: Catamaran; SSB; Longitudinal Motion; Seakeeping; CFD; Model test

\begin{abstract}
In order to improve seakeeping performance of the slender catamaran, Semi Submerged Bow (SSB) anti-pitching techniques is applied. Based on CFD method to forecast the longitudinal motion of the original catamaran (without SSB) in regular waves, and the calculation results and the ship model test results were compared, results show that the numerical method can effectively predict the longitudinal motion of the slender catamaran in regular waves. Semi Submerged Bow is added to the bottom of two demi-hull respectively, and the longitudinal motion in regular waves is predicted. The results show that the longitudinal motion amplitude of catamaran with SSB is smaller than the catamaran without SSB. The installation of SSB reasonably can effectively improve the seakeeping performance of the catamaran.
\end{abstract}

\section{Introduction}

As a high-performance ship, catamaran has a spacious deck area and good stability, smaller swing and tilt magnitude, consumes less power, low fuel cost and high economical compared with the monohull. The concept of SSB was first proposed by the Japanese designer in the 1980s, was applied to passenger vessel ship through demonstration and testing in 1986s, the effect was very satisfactory. Huang et al. studied the round bilge hull with SSB, the results showed that SSB could effectively improve seakeeping performance of the hull [1]. Cai et al. has long been committed to researching antiroll appendage, and developed a monomer drainage composite ship which bow installed SSB and delta wing type fin in the 1990s, the calculations and tests showed that the longitudinal movement response of the ship in waves was improved and the seakeeping performance was significantly improved [2]. This paper presents a catamaran hull with SSB and explores its advantages.

\section{Numerical Methods}

Conservation of mass and momentum conservation equations were [3-4]:

$$
\begin{aligned}
& \frac{\partial p}{\partial t}+\frac{\partial\left(\rho u_{i}\right)}{\partial x_{i}}=0 \\
& \frac{\partial\left(\rho u_{i}\right)}{\partial t}+\frac{\partial}{\partial x_{i}}\left(\rho u_{i} u_{j}\right)=-\frac{\partial p}{\partial x_{i}}+\frac{\partial}{\partial x_{j}}\left(\mu \frac{\partial u_{i}}{\partial x_{j}}-\rho \overline{u_{i}^{\prime} u_{j}^{\prime}}\right)+S_{i}
\end{aligned}
$$

Where $u_{i}$ and $u_{j}$ are the mean of velocity components $(i, j=1,2,3), P$ is the mean of pressure; $\rho$ is the fluid density; $\mu$ is dynamic viscosity coefficient; $\rho \overline{u_{i}^{\prime} u_{j}^{\prime}}$ is the Reynolds stress; $S_{i}$ is generalized source term of momentum equation.

Turbulence model uses SST $k$ - $\omega$ model, which is the shear stress transport model [5], the equations $k$ and $\omega$ are as follows:

* Corresponding author, email: zhuangjiayuan@ hrbeu.edu.cn 


$$
\begin{aligned}
& \frac{\partial(\rho k)}{\partial t}+\frac{\partial}{\partial x_{i}}\left(\rho k u_{i}\right)=\frac{\partial}{\partial x_{i}}\left(\Gamma_{k} \frac{\partial k}{\partial x_{i}}\right)+G_{k}-Y_{k} \\
& \frac{\partial(\rho \omega)}{\partial t}+\frac{\partial}{\partial x_{i}}\left(\rho \omega u_{i}\right)=\frac{\partial}{\partial x_{i}}\left(\Gamma_{\omega} \frac{\partial \omega}{\partial x_{i}}\right)+G_{\omega}-Y_{\omega}+D \omega
\end{aligned}
$$

Where $G_{k}$ and $G_{\omega}$ are turbulent kinetic energy term expressed by the velocity gradient produced, $\Gamma_{k}$ and $\Gamma_{\omega}$ are $k$ and $\omega$ the convection term; $Y_{k}$ and $Y_{\omega}$ are $k$ and $\omega$ turbulent diffusion.

Free surface simulation uses the VOF method [6-7]. Assuming the fluid density of the entire flow region is constant $\rho_{0}$, the air density is set to zero, and the fluid volume parameters are $F=$ $\rho / \rho_{0}$, the convection equation for $F$ is:

$$
\frac{\partial F}{\partial t}+\vec{u} \cdot \nabla F=0
$$

If a grid cell in a moment $F=1$, the grid cell fills with a specified fluid; If $F=0$, the grid cell fills with other fluids. If $0<F<1$, the grid cell includes the free surface.

\section{Model Test and Accuracy Verification}

The ship model tests conducted in towing tank at Harbin Engineering University, the tank length is $108 \mathrm{~m}$, width is $7 \mathrm{~m}$, depth is $3.5 \mathrm{~m}$, maximum speed of trailer is $6.5 \mathrm{~m} / \mathrm{s}$, wave period of wave machine is $0.4-4.0 \mathrm{~s}$, maximum wave height of wave machine is $0.4 \mathrm{~m}$, the data acquisition system is the DHDAS_5920 dynamic signal acquisition and analysis system, the main test instruments are a force balance, gyroscope, heave rods, accelerometer, thermometer. Tests using airworthiness instrument by freely dragging measure resistance, trim, heave and other parameters. Models make of FRP composite materials (as Figure 1). Model test wave height is $0.04 \mathrm{~m}$, regular wave lengths including $2.24 \mathrm{~m}, 3.14 \mathrm{~m}, 3.59 \mathrm{~m}, 4.03 \mathrm{~m}, 4.48 \mathrm{~m}, 4.93 \mathrm{~m}, 5.38 \mathrm{~m}, 5.83 \mathrm{~m}, 6.72 \mathrm{~m}, 7.84 \mathrm{~m}$, speeds is $2.18 \mathrm{~m} / \mathrm{s}$ and $2.91 \mathrm{~m} / \mathrm{s}$. The main scale parameters are shown in Table 1, lines plan is shown in Figure 2.

Table 1 Main dimension of the model without SSB

\begin{tabular}{c|c}
\hline Main dimension & Model without SSB \\
\hline Overall length $L(\mathrm{~mm})$ & 4480 \\
\hline Overall width $b(\mathrm{~mm})$ & 956 \\
\hline Demihull spacing $k(\mathrm{~mm})$ & 715 \\
\hline Designed displacement $\Delta(\mathrm{kg})$ & 250 \\
\hline Designed draft $t(\mathrm{~mm})$ & 152 \\
\hline Water line length $L_{W L}(\mathrm{~mm})$ & 4224 \\
\hline Longitudinal center of gravity $X_{g}(\mathrm{~mm})$ & 1994 \\
\hline Vertical center of gravity $Z_{g}(\mathrm{~mm})$ & 155 \\
\hline \\
\hline
\end{tabular}

Fig. 1 Model test of ordinary catamaran

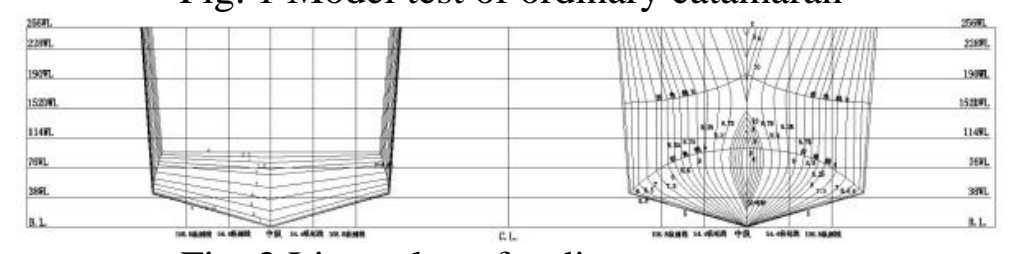

Fig. 2 Lines plan of ordinary catamaran 
Meshing uses unstructured grid technology, computational domain is a cuboid, the range of longitudinal extends forward doubled length from the bow, back 2.5 times length from the stern, the width is two times of length, the vertical direction extends length down from waterline, 0.5 times length up from waterline. Domain grid and surface mesh of the model without SSB are shown in Figure 3.

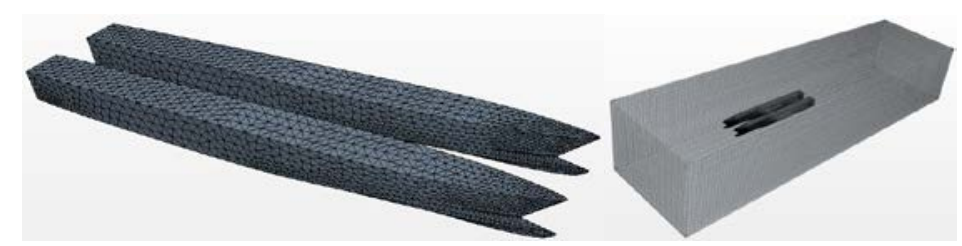

Fig. 3 The surface mesh and computed domain of model without SSB

Boundary conditions: the import, up and down of the computational domain are defined as the entrance of rate, hull surface is defined as the no-slip boundary, and the outlet of the computational domain is defined as a pressure outlet.

This paper based on VOF wave model and first-order wave forms calculated the response of longitudinal movement in regular waves for head sea when speed at 2.18 and $2.91 \mathrm{~m} / \mathrm{s}$, amplitude at $0.02 m$, wavelength under 2.24, 3.14, 3.59, 4.03, 4.48, 4.93, 5.38, 5.83, 6.72, 7.84m.

The calculated results and experimental results in regular waves for head sea under speed of 2.91 $\mathrm{m} / \mathrm{s}$ are compared in terms of the amplitude-frequency response functions of pitch, heave, and acceleration, as showed in Figure 4. The abscissa of each graph is the encountered frequent $\omega_{e}$, in $\mathrm{rad} / \mathrm{s}$, while the ordinate in each case is the amplitude-frequency response function. For pitch, it is the amplitude of the pitch to the wave amplitude multiplied by $k\left(\theta_{a} / k \zeta_{a}\right), k=2 \pi / \lambda$; for heave, the ordinate is the amplitude of the heave to wave ratio $\left(z_{a} / \zeta_{a}\right)$; and for acceleration, it is the amplitude of the acceleration at the center of gravity multiplied by the model length, divided by the gravitational acceleration constant multiplied by the amplitude of the wave ratio $\left(a_{m} L_{m} / g \zeta_{a}\right)$.

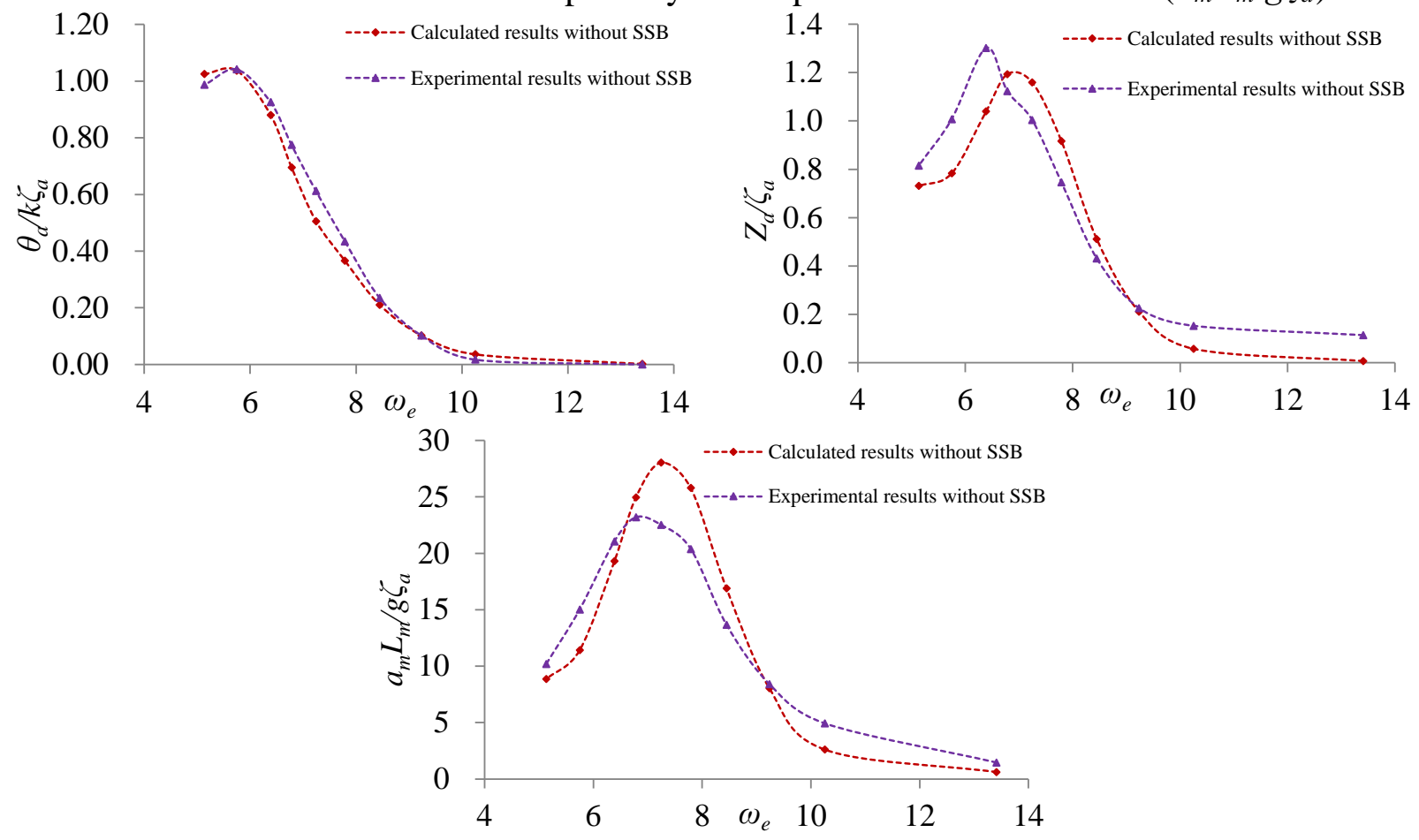

Fig. 4 Pitching, heaving, and acceleration comparison at speed of $2.91 \mathrm{~m} / \mathrm{s}$

The comparative analysis of numerical and experimental results indicates that using CFD method to forecast pitching, heaving and acceleration of the catamaran in regular waves for head sea is feasible, and satisfies the engineering accuracy.

\section{Numerical Results of the Catamaran with SSB}

Semi-submersible could significantly improve seakeeping performance by generating opposite 
viscous damping torque of vessel movement, thereby. Based on conventional catamaran, this paper installed SSB in the bow, researched the performance of longitudinal movement in regular waves for head sea using numerical simulation methods, and made a comparative analysis between the two ships. Longitudinal section of SSB uses NACA airfoil, cross-section uses the elliptical, the volume is the $1.7 \%$ of the total drainage vessel. Dimensions of the SSB are given in Table 2. Lines plan of the model with SSB is shown in Figure 5. Domain grid and surface mesh of the model with SSB are shown in Figure 6.

Table 2 Main dimension of SSB

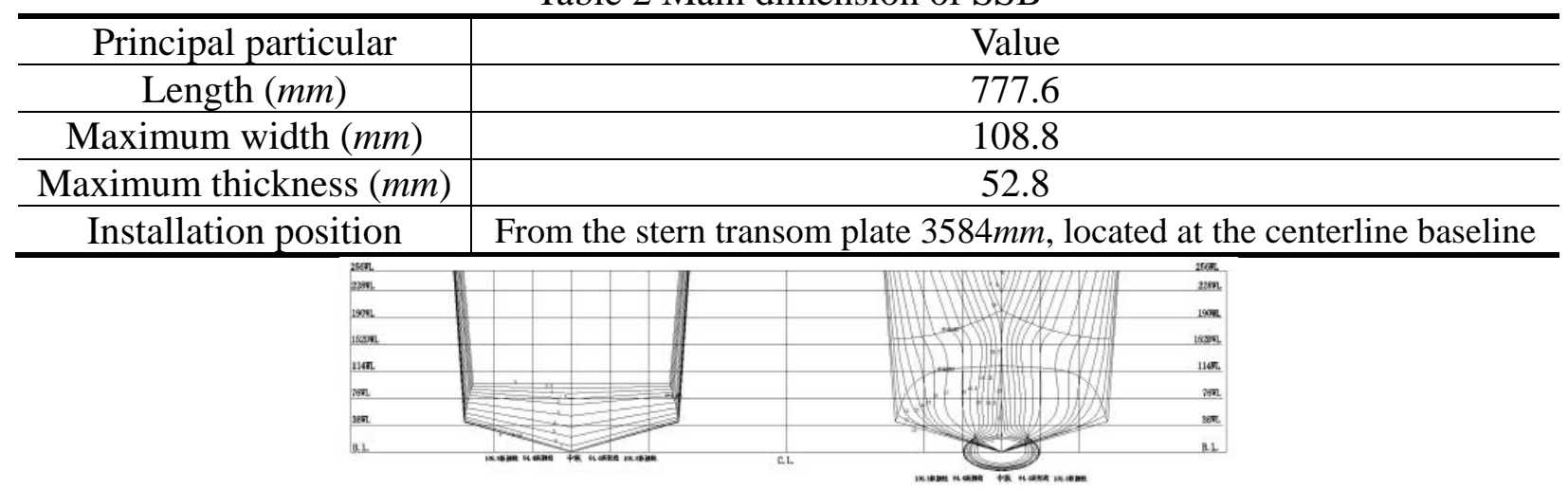

Fig. 5 Lines plan of the model with SSB

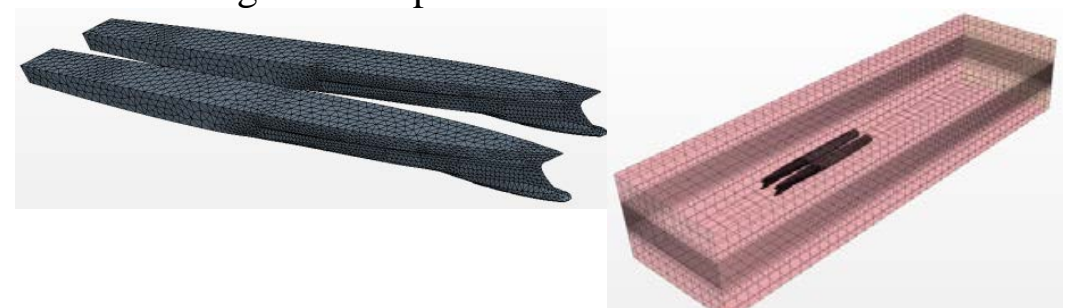

Fig. 6 The surface mesh and computed domain of the model with SSB

The calculated results of two forms in regular waves under different speed are compared in Figures 7-8.

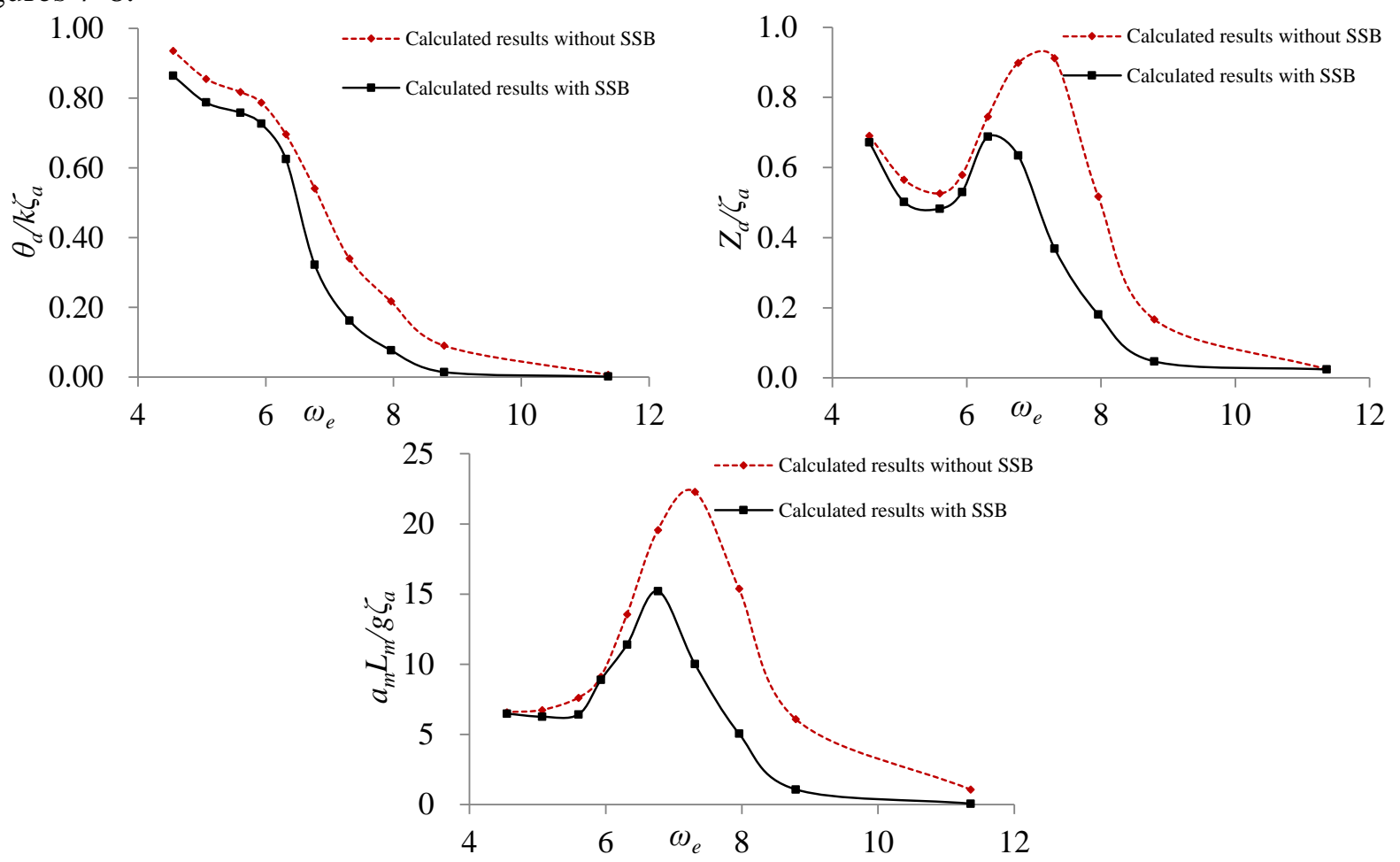

Fig. 7 Pitching, heaving, and acceleration of different models at speed of $2.18 \mathrm{~m} / \mathrm{s}$ 


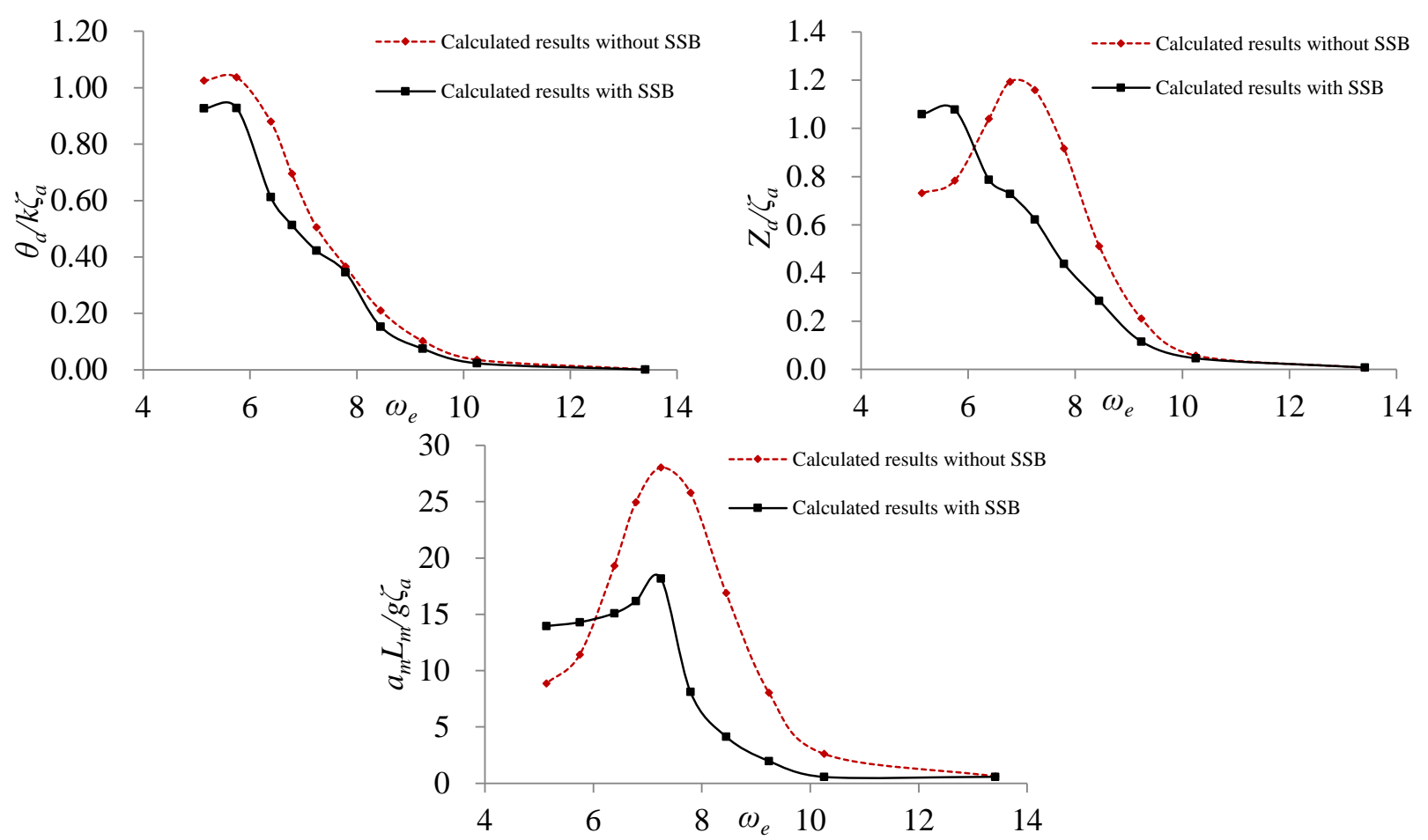

Fig. 8 Pitching, heaving, and acceleration of different models at speed of $2.91 \mathrm{~m} / \mathrm{s}$

Numerical results show that the catamaran with SSB has a better longitudinal motion performance than ordinary, can effectively reduce the pitch, heave and acceleration amplitude, antiroll effect under various speed are shown in Table 3.

Table 3 Antiroll efficiency

\begin{tabular}{c|c|c}
\hline Speed $(\mathrm{m} / \mathrm{s})$ & 2.18 & 2.91 \\
\hline Pitch effect & $-35.57 \%$ & $-25.29 \%$ \\
\hline Heave effect & $-26.53 \%$ & $-17.70 \%$ \\
\hline Acceleration effect & $-37.46 \%$ & $-31.57 \%$ \\
\hline
\end{tabular}

\section{Conclusion}

This paper studied numerical prediction and experiment of pitching and heave motion about ordinary and semi-submersible catamaran in regular waves for head sea based on numerical methods of viscous flow theory, unstructured grids and a first-order wave form, made comparative analysis of the calculated and experimental values showing that forecast values are in good agreement with the experimental results, indicated that it is feasible to forecast the seakeeping of SSB catamaran ship by numerical simulation method.

The catamaran with SSB can effectively improve the longitudinal motion than the original hull. The effects of pitching and acceleration are obvious, the magnitude of improvement in speed 2.18 $\mathrm{m} / \mathrm{s}$ is $-35.57 \%$ and $-37.46 \%$ respectively.

\section{Acknowledgement}

In this paper, the research was sponsored by the National Natural Science Foundation of China (No. 51409069 and No. 51409054), and Fundametal Research Funds for the Central Universities (HEUCF150118).

\section{References}

[1] Huang Dingliang, Ai Zhidan, Xing Dianlu. The study on seakeeping of semi-submersible properties. Chinese shipbuilding, 1992 (1). 
[2] Cai Xin Gong, Li Jide, Liu Yadong, Wang Jianfang. Motion prediction on the ship of Installation anti-pitching combination possessed [J]. Hydrodynamics Research and Development, 2003,18 (2) :148-155.

[3] Wu Ziniu. Basic principles of computational fluid dynamics [M]. Beijing: Science Press, 2001.

[4] WangFujun. computational fluid dynamics analysis [M]. Beijing: Tsinghua University Press, 2006.

[5] Wang Qiang. Based on SSTk-turbulence model numercal study of high-rise buildings shape coefficient [J]. Shenyang Jianzhu University, 2012,5 (3).

[6] Nichols B D, Hirt C W. SOLA-VOF:A solution algorithm for transient fluid flow with multiple free-bound Alamos Scientific Laboratory report,1980, LA-8355.

[7] Li Shaowu, Yin Zhenjun. Numerical solution of NS equations and review the application of the wave dynamics [J]. Marine Science Bulletin, 2004,8. Vol.23 (4). 